\title{
Evidence that mastitis can cause pregnancy loss in dairy cows: A systematic review of observational studies
}

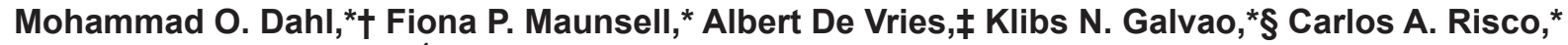 \\ and Jorge A. Hernandez*1 \\ *Department of Large Animal Clinical Sciences, College of Veterinary Medicine, University of Florida, Gainesville 32611-0910 \\ †Department of Medicine and Preventive Medicine, College of Veterinary Medicine, University of Mosul, Mosul, Iraq 41002 \\ łDepartment of Animal Sciences, College of Agriculture and Life Sciences, University of Florida, Gainesville 32611-0910 \\ §D. H. Barron Reproductive and Perinatal Biology Research Program, University of Florida, Gainesville 32610
}

\begin{abstract}
The objective of this study was to conduct a systematic review to identify and assess evidence and knowledge gaps in published observational studies that have investigated the relationship between mastitis and pregnancy loss (PL) in dairy cows. PubMed and ScienceDirect were used to search pertinent peer-reviewed research reports of interest. Screening of research reports was conducted at 3 levels: titles, abstracts, and full-text articles. The search identified 651 records for initial screening. The final screening process identified 8 qualified articles for review after removing 10 duplicate records, 582 titles, 31 abstracts, and 20 full-text articles. Two studies produced strong epidemiologic evidence indicating that (1) exposure to clinical mastitis during early gestation (first $45 \mathrm{~d}$ of gestation) is associated with subsequent PL during the following 90 $\mathrm{d}$; and (2) subclinical mastitis 1 to $30 \mathrm{~d}$ before artificial insemination (AI) is associated with subsequent PL at 35 to $41 \mathrm{~d}$ of gestation. An additional study showed that exposure to clinical mastitis during early lactation in combination with low body condition can increase the risk of PL in dairy cows; however, the interaction effect between clinical mastitis and low body condition on PL was considered weak. Four other studies produced inconclusive evidence indicating that mastitis is a predisposing factor for PL in dairy cows, as the exposure risk period for mastitis overlapped with the follow-up period for diagnosis of PL in dairy cows. Finally, one study failed to identify a relationship between mastitis and PL in dairy cows. Further research is needed to (1) support the hypothesis that mastitis in combination with low body condition score (or other exposure fac-
\end{abstract}

Received February 10, 2017.

Accepted June 9, 2017.

${ }^{1}$ Corresponding author: hernandezja@ufl.edu tors) can increase the risk of PL, (2) compare the effect of clinical versus subclinical mastitis on PL, (3) compare the effect of mastitis before breeding and during gestation on PL, and (4) compare the effect of mastitis on PL in dairy cows during different lactations.

Key words: pregnancy loss, mastitis, dairy, systematic review

\section{INTRODUCTION}

Pregnancy loss (PL) has been defined as the loss of pregnancy in cows that were first confirmed pregnant at about 30 to $50 \mathrm{~d}$ after $\mathrm{AI}$ and later exhibited visual signs of abortion or were declared not pregnant during reconfirmation of pregnancy (Risco et al., 1999; Chebel et al., 2004; Moore et al., 2005). Pregnancy loss impairs reproductive performance in dairy cows, thus affecting the profitability of dairy farms. Economic losses due to PL have ranged between $\$ 555$ and $\$ 2,333$ as a result of repeat breeding, extended calving interval, labor and medical costs, decreased milk yield, and culling of dairy cows (De Vries, 2006; Lee and Kim, 2007). In the United States, prevalence of PL in dairy cows has varied from 6 to 39\% (Risco et al., 1999; Chebel et al., 2004; Moore et al., 2005).

Embryonic mortality and PL can be linked to mastitis as a function of endotoxins or inflammatory and immune responses. Inducing clinical mastitis with Escherichia coli, Klebsiella pneumoniae, or Streptococcus uberis in dairy cows increased SCC in milk (Paape et al., 2002; Persson Waller et al., 2003), the concentration of $\mathrm{PGF}_{2 \alpha}$ in milk and plasma (Cullor, 1990; Hockett et al., 2000), and the activity of pro-inflammatory cytokines, particularly tumor necrosis factor (TNF)- $\alpha$, IL-1 $\beta$, and IL-8 in milk, lymph, and blood (Shuster et al., 1993; Rainard and Paape, 1997; Hoeben et al., 2000; Paape et al., 2002; Persson Waller et al., 2003; Rambeaud et al., 2003). Cytokines have cytotoxic activity on the corpus luteum (Schams and Berisha, 2004) and can increase $\mathrm{PGF}_{2 \alpha}$ concentration (Townson 
and Pate, 1994), resulting in luteolysis and termination of pregnancy. Endotoxins and cytokines $(\mathrm{TNF}-\alpha)$ can impair early embryonic development, affecting embryo survival (Soto et al., 2003; Hansen et al., 2004).

Several observational studies have reported that clinical or subclinical mastitis is a predisposing factor for PL in dairy cows (Risco et al., 1999; Chebel et al., 2004; Santos et al., 2004; McDougall et al., 2005; Moore et al., 2005; Pinedo et al., 2009; Hernandez et al., 2012). In 2 studies, the odds of PL were 2.7 or 2.8 times higher in cows affected with clinical mastitis during early gestation compared with cows without clinical mastitis (Risco et al., 1999; Chebel et al., 2004). In 2 other studies, exposure to clinical mastitis any time during lactation was associated with an increased risk of PL in dairy cows (Santos et al., 2004; McDougall et al., 2005). In 2 additional studies, the odds of PL were $>3.5$ times higher in cows affected with subclinical mastitis before gestation (Moore et al., 2005) or 1.2 times higher in cows with subclinical mastitis during early gestation (Pinedo et al., 2009) compared with cows without mastitis. A seventh study identified an interaction effect between clinical mastitis during early lactation and low body condition at 70 DIM on PL in dairy cows (Hernandez et al., 2012). Finally, a study by López-Gatius et al. (2002) failed to identify a relationship between clinical mastitis and PL in dairy cows during the first 38 to $90 \mathrm{~d}$ of gestation. Although published observational studies have provided different levels of evidence indicating that mastitis can cause PL in dairy cows, most of these studies used nonobjective research methods and failed to establish a temporal relationship between mastitis and PL in dairy cows, making their study results inconclusive. To our knowledge, a systematic review that has carefully examined the evidence of association between mastitis and PL in dairy cows produced by observational studies has not been published. Therefore, our objective was to conduct a systematic review to identify and assess the evidence and knowledge gaps in published observational studies that have investigated the relationship between mastitis and PL in dairy cows.

\section{MATERIALS AND METHODS}

A systematic review for epidemiologic studies that examined mastitis as a predisposing factor for PL in dairy cows was conducted according to Preferred Reporting Items for Systematic reviews and MetaAnalyses (PRISMA) statement (Moher et al., 2009). In this study, PL was defined as cows that were declared pregnant at about d 30 after AI and later exhibited visual signs of abortion or were diagnosed as not pregnant via ultrasound or rectal palpation.

\section{Eligibility Criteria}

Observational studies written in English that presented original data and were published in peerreviewed journals were considered for inclusion. Narrative reviews, meta-analyses, or studies published only as abstracts were excluded. Characteristics of studies included in this review were identified according to the PICOS approach (P: population; I: intervention (or exposures); C: comparators; O: outcome; and S: study designs; Liberati et al., 2009) and included (1) study population: dairy cows during different parities from dairy herds inside or outside the United States; (2) exposure of interest: clinical or subclinical mastitis, provided that clinical mastitis was diagnosed by detecting abnormal changes in the milk or udder, and subclinical mastitis was identified by applying diagnostic tests to the milk, such as SCC, assuming that cows with mastitis were identified equally accurately in all studies; (3) comparators: dairy cows that had not been exposed to clinical or subclinical mastitis; (4) outcome of interest: PL, which was identified as declared pregnant cows that exhibited visual signs of abortion or were diagnosed not pregnant during second or later pregnancy diagnosis, where first pregnancy diagnosis in commercial herds is usually performed around d 30 after AI; and (5) study design: observational studies targeted examining the association between PL and exposure to mastitis alone or in combination with other exposure factor(s).

\section{Information Sources and Selection Process}

Two online search engines, PubMed and ScienceDirect, identified as high-quality scientific search engines (Samadzadeh et al., 2013) were used to track pertinent peer-reviewed research reports. The search was initially conducted on February 21, 2014, and then updated on April 3, 2016. Keywords used were mastitis, pregnancy, abortion, dairy, cow, and cattle. Selected keywords were entered in the search box as a phrase where each word was followed by comma and one space as follows: "mastitis, pregnancy, abortion, dairy, cow, cattle". The PubMed database was selected from "database selection menu" located on the left of the search box on PubMed's home page display (https://www.ncbi.nlm .nih.gov/pubmed). In addition, no filters were applied in either PubMed or ScienceDirect. Duplicate records from both PubMed and ScienceDirect were excluded. Screening was conducted at 3 levels: titles, abstracts, 
and full-text articles to determine the final number of articles qualified for review (Figure 1).

\section{Data Extraction Process and Items}

One author (MOD) independently extracted data from included studies, and a second author (JAH) checked the extracted data. Data included the following: study type, study sample size, type of mastitis (clinical or subclinical mastitis), risk period for mastitis (before breeding, between breeding and pregnancy diagnosis, after pregnancy diagnosis, whole lactation period), follow-up period for PL, effect estimates (odds ratio, risk ratio, hazard ratio, proportions, or least squares means) with $95 \%$ confidence intervals if applicable, and covariates included in the final models (Table 1).

\section{Assessment of Qualified Studies}

Methods for assessing potential bias were examined based on research methods and techniques used in selected studies. First, an assumption was that the strength of evidence was highest $(+++; 3$ points $)$ in well-designed cohort studies compared with casecontrol studies $(++)$ or cross-sectional studies $(+)$. In addition, 2 key issues of interest were selection bias (potential misclassification of PL as an outcome) and exposure bias (potential misclassification of mastitis),

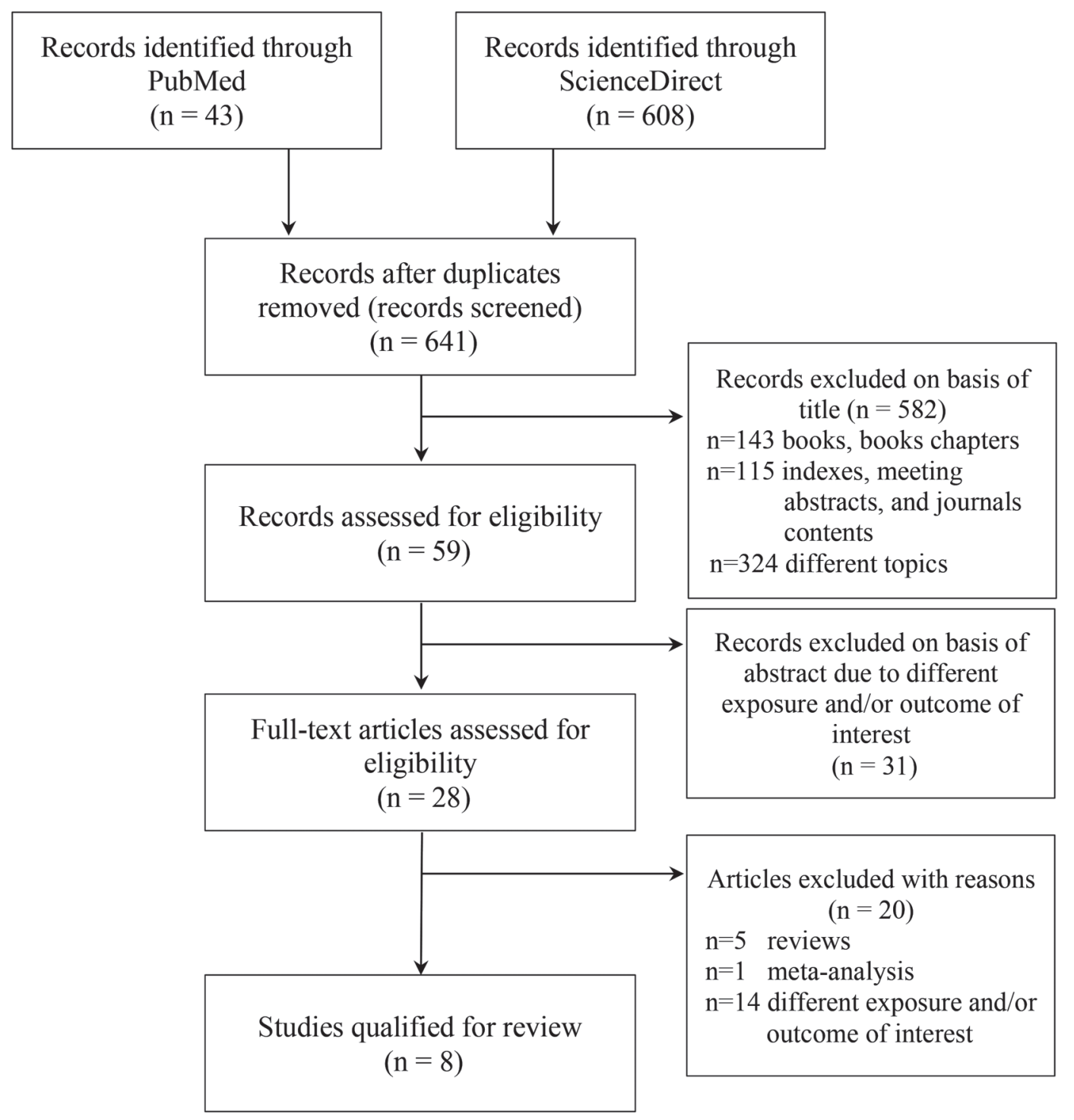

Figure 1. Flow diagram of article selection process applied in the systematic review for epidemiology of pregnancy loss attributable to mastitis in dairy cows. Key words of mastitis, pregnancy, abortion, dairy, cows, and cattle were used on April 3, 2016. 
MASTITIS AND PREGNANCY LOSS: SYSTEMATIC REVIEW

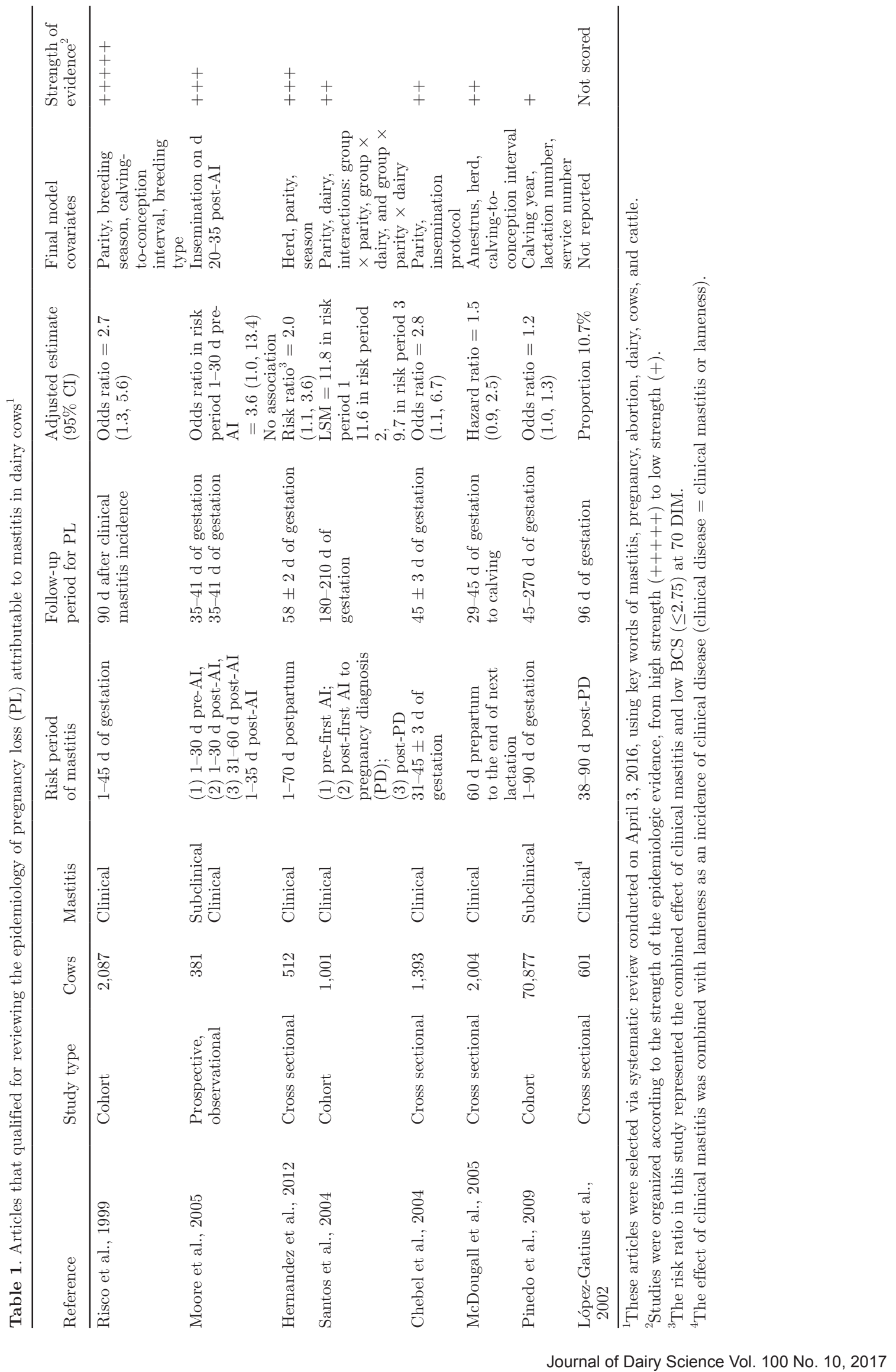


particularly if the study of interest carefully examined (yes, no) the temporal relationship between mastitis as a predisposing factor for PL in study cows. For example, we assigned 2 points $(++)$ to studies where risk of selection bias and exposure bias was considered negligible; one point $(+)$ to studies with evidence that selection bias or exposure bias was present or not negligible; and zero points to studies with evidence that both selection bias and exposure bias were present. Thus, the maximum number of points assigned to each study was 5 .

\section{RESULTS AND DISCUSSION}

A total of 43 and 608 records were initially identified using PubMed and ScienceDirect, respectively, for a total of 651 records. Eight articles qualified for review after removing 10 duplicate records, 582 titles, 31 abstracts, and 20 full-text articles (Figure 1). The selected articles included 8 observational studies that examined the relationship between PL and mastitis (clinical or subclinical) in dairy cows (Table 1). These studies produced different levels of strength of evidence. Most of them $(5 / 8)$ failed to establish a temporal relationship between mastitis and PL. We discuss each of these studies in this report, starting with the highest strength of evidence and ending with the lowest.

In the study by Risco et al. (1999) (strength of evidence: +++++ ), cows affected with clinical mastitis during the first $45 \mathrm{~d}$ of gestation had a 2.7 times higher risk of PL within the following $90 \mathrm{~d}$ after diagnosis of mastitis than cows without mastitis [odds ratio (OR) $=2.7 ; 95 \% \mathrm{CI}=1.3,5.6]$. Risco et al. (1999) explained that PL was attributable to clinical mastitis due to pyrexia, activity of pro-inflammatory cytokines, or the luteolytic action of $\mathrm{PGF}_{2 \alpha}$, which is released as a part of the inflammatory response. The investigation was designed as a cohort study, where the odds of PL were compared in cows with or without previous exposure to clinical mastitis. In that study, selection bias was negligible. Cows were enrolled when declared pregnant by rectal palpation on d 42 to 49 of gestation. In that study, PL was determined when cows exhibited visual signs of abortion or returned to service after being confirmed pregnant, or in pregnant cows that were found open during reconfirmation of pregnancy at the time of dry-off. In the study herd, cows confirmed pregnant were examined for estrus and monitored for signs of abortion on a daily basis. Cows detected in estrus that were previously confirmed pregnant or those suspected of having an abortion were palpated by a veterinarian within $7 \mathrm{~d}$ of either of these 2 observations. In addition, exposure bias was considered negligible. The study used a standardized definition for clinical mastitis (e.g., cows were considered to have clinical mastitis if abnormalities of the milk or any quarter were detected between successful breeding and pregnancy examination by milking personnel). Finally, in this study, the temporal relationship between mastitis and PL was carefully examined. The exposure risk period for mastitis did not overlap with that for the follow-up period of PL.

In the study by Moore et al. (2005) $(+++)$, the odds of PL during 35 to $41 \mathrm{~d}$ of gestation were 3.6 times higher in cows with DHIA test SCS $>4.5$ during the 30 $\mathrm{d}$ before AI compared with cows without mastitis (OR $=3.6 ; 95 \% \mathrm{CI}=1.0,13.4)$. Although an explanation for this observed association was not reported in that study, we offer the following hypothetical explanation: subclinical mastitis can trigger a systemic inflammatory response that can affect follicular growth, oocyte quality, and embryo survival (Britt, 2008; Ribeiro et al., 2016). In Moore et al. (2005) study, 381 pregnant cows were enrolled at the time of AI. The investigation was designed as an observational study. Selection of study cows was conducted by using a cross-sectional approach, where all study cows diagnosed pregnant during a 2-mo period were considered for inclusion. Exposure status to mastitis and PL status were not known when cows were selected for inclusion. Selection bias was negligible, as diagnosis of PL was conducted using standard methods. Cows declared pregnant via transrectal ultrasound on d 27 or 28 after AI were investigated for PL by rectal palpation (absence of amniotic vesicle) on d 35 to 41 after AI. Exposure bias was negligible because diagnosis of mastitis was based on DHIA SCS data. In addition, the temporal relationship between mastitis and PL was well examined; the risk period for mastitis did not overlap with the follow-up period for PL.

In the study by Hernandez et al. (2012) (+++), exposure to clinical mastitis during early lactation in combination with low body condition $(\mathrm{BCS} \leq 2.75)$ at 70 DIM increased the risk of PL in dairy cows [risk ratio $(\mathbf{R R})=2.0 ; 95 \% \mathrm{CI}=1.1,3.6)$. In that study, the authors explained that clinical mastitis during the first 10 to 50 DIM can induce prolonged negative energy balance in cows leading to losses of BW and body condition days before or after insemination. In that study, although the observed combined effect between clinical mastitis and low body condition on PL was statistically significant, the interaction effect, if present, was considered weak; the expected joint effect of mastitis and low body condition on PL $(\mathrm{RR}=1.4)$ was close to the observed combined effect of those 2 exposure factors on $\mathrm{PL}(\mathrm{RR}=2.0)$. The investigation was designed as a cross-sectional study. The frequency of clinical mastitis, low body condition, and other investigated exposure factors was compared between 88 
cows diagnosed with PL $60 \mathrm{~d}$ after first postpartum TAI and 424 cows that were not. Selection bias was negligible; PL was determined when study cows were diagnosed with absence of embryonic vesicle via rectal palpation after $28 \mathrm{~d}$ of pregnancy diagnosis. Exposure bias was considered negligible. All study cows were examined daily for clinical mastitis by farm personnel during each milking during the first 70 DIM. A case of mastitis was characterized by the presence of abnormal milk or signs of inflammation in one or more quarters, or both. In addition, although the investigation was designed as cross-sectional study, all cows with mastitis were affected with the disease before breeding.

In Santos et al. (2004) study $(++)$, incidence of PL was higher in cows affected with clinical mastitis (9.7 to $11.8 \%$ ) than in cows without clinical mastitis (5.8\%). In that study, clinical mastitis was investigated during 3 exposure periods: (1) before first postpartum AI; (2) between first postpartum AI and pregnancy diagnosis (35 to $48 \mathrm{~d}$ after $\mathrm{AI}$ ); and (3) after study cows were diagnosed pregnant (35 to $48 \mathrm{~d}$ after AI until 180 to $210 \mathrm{~d}$ of gestation or end of current lactation). Santos et al. (2004) explained that inflammatory response due to mastitis can cause luteolysis as a function of $\mathrm{PGF}_{2 \alpha}$ release, thus affecting oocyte growth during the first and second mastitis exposure periods, and terminating pregnancy during the third period. The investigation was designed as a cohort study. Selection bias was not negligible because the research methods used did not include a definition of PL in study cows. Although diagnosis of clinical mastitis used sound methods, exposure bias was not negligible. All study cows were examined for signs of clinical mastitis at every milking by the herd personnel immediately before milking. Clinical mastitis cases were characterized by the presence of abnormal milk or by signs of inflammation in one or more quarters, and were treated by intramammary infusion of antibiotics according to treatment protocols established by the attending veterinarian. Finally, although the temporal relationship between mastitis and PL was well examined during the first and second mastitis exposure periods, it was not during the third period because the exposure period for mastitis (35 to $48 \mathrm{~d}$ after AI until 180 to $210 \mathrm{~d}$ of gestation or end of current lactation) potentially overlapped with the follow-up period for PL.

In the Chebel et al. (2004) study $(++)$, the odds of PL were 2.8 times higher in cows affected with clinical mastitis during the first $45 \mathrm{~d}$ of gestation, compared with cows without clinical mastitis $(\mathrm{OR}=2.8 ; 95 \%$ $\mathrm{CI}=1.1,6.7 ; P=0.02)$. The authors did not offer an explanation for the observed association between mastitis and PL in study cows, except that previous studies had identified an association between mastitis and
PL in dairy cows. The investigation was designed as a cross-sectional study. The frequency of clinical mastitis (and other exposure factors) was compared between 183 cows diagnosed with PL and 1,282 cows that were not. Selection bias was considered negligible; PL was determined when a cow was diagnosed as pregnant (31 $\pm 3 \mathrm{~d}$ after AI via transrectal ultrasonography) and was not confirmed pregnant by palpation per rectum 14 d later. Exposure bias was considered not negligible. All cows were examined for signs of clinical mastitis by herd personnel immediately before every milking. Clinical mastitis cases were characterized by the presence of abnormal milk or by signs of inflammation in one or more quarters. However, the temporal relationship between mastitis and PL was not well examined, as the risk period for mastitis was the same for the follow-up period of PL (1 to $45 \mathrm{~d}$ after AI).

In the McDougall et al. (2005) study $(++)$, the risk of PL was higher in cows with clinical mastitis (hazard ratio $=1.5 ; 95 \% \mathrm{CI}=0.9,2.5 ; P=0.07)$ than in cows without clinical mastitis. The authors explained that IMI can increase uterine sensitivity to $\mathrm{PGF}_{2 \alpha}$ and prostaglandin metabolite levels in blood following exposure to oxytocin, leading to subsequent PL in dairy cows. The investigation was designed as a crosssectional study. The frequency of clinical mastitis (and other exposure factors) was compared among 128 cows diagnosed with PL and 1,876 cows that were not. Selection bias was considered negligible. Cows examined for pregnancy diagnosis on d 29 to 45 after breeding and 3 to 4 times later during gestation. Pregnancy loss was determined when a cow diagnosed as pregnant was later found either not pregnant or at a stage of gestation not compatible with the conception date determined previously. Exposure bias was considered not negligible. Herd owners diagnosed clinical mastitis in study cows based on the presence of gross abnormalities of the udder (heat, swelling or pain), presence of changes in composition of the milk (clots, blood), or both. However, the temporal relationship between mastitis and PL was not well examined. The exposure risk period for clinical mastitis extended from $60 \mathrm{~d}$ before calving to the end of the current lactation. Thus, the risk period for mastitis may have overlapped with the follow-up period for PL in study cows.

In the Pinedo et al. (2009) study (+), the odds of PL were 1.2 times greater in cows affected with subclinical mastitis (SCS $\geq 4.5$ ) during the first $90 \mathrm{~d}$ of gestation compared with cows without subclinical mastitis (OR $=1.2 ; 95 \% \mathrm{CI}=1.0$ to 1.3 ). The authors did not offer an explanation for the observed association between subclinical mastitis and PL in study cows, except that previous studies had identified an association between mastitis and PL in dairy cows. The investigation was 
reportedly designed as a historic cohort study. However, study pregnant cows were not enrolled based on exposure (yes, no) to subclinical mastitis and followed over time for diagnosis of PL. Rather, all pregnant cows selected for inclusion $(\sim 70,000)$ were classified as affected with PL (yes, no) and mastitis (yes, no), a sampling approach that is more aligned with a cross-sectional study than a cohort study. In that study, the frequency of subclinical clinical mastitis (and other exposure factors) was compared between cows with or without PL. Selection bias was considered not negligible. Although pregnancy loss was defined as the loss of the conceptus after $45 \mathrm{~d}$ of gestation until $270 \mathrm{~d}$ of gestation, methods used for diagnosis of PL (ultrasonography, palpation per rectum, cows confirmed pregnant were examined for estrus and monitored for signs of abortion on a daily basis) were not reported. Exposure bias was not negligible. Cows with subclinical mastitis were those affected with high natural log-transformed log linear score (LNSCC) $\geq 4.5$ during the first $90 \mathrm{~d}$ of gestation. However, the temporal relationship between subclinical mastitis and PL was not well examined, as the risk period for mastitis (1 to $90 \mathrm{~d}$ of gestation) overlapped with that for the follow-up period of PL (45 to $270 \mathrm{~d}$ after AI).

Finally, the study by López-Gatius et al. (2002) examined the relationships between several exposure factors, including clinical mastitis, and PL in dairy cows during the first 38 to $90 \mathrm{~d}$ of gestation. In this study, mastitis was not associated with PL in study cows. The investigation was designed as a cross-sectional study. The frequency of clinical mastitis (or lameness) and other exposure factors were compared in 64 cows diagnosed with PL and 537 cows that were not. Selection bias was negligible. Pregnancy diagnosis was performed by ultrasonography between 38 to $44 \mathrm{~d}$ after AI. Pregnancy loss was determined when study cows were diagnosed as nonpregnant via rectal palpation between 90 and 96 d after AI. Exposure bias was considered not negligible. Clinical mastitis was not defined, except that cases of mastitis were those diagnosed following pregnancy diagnosis. Number of cows affected with mastitis was not reported. In the analysis, cases of mastitis or lameness were combined into one exposure variable of "clinical disease." Overall, in that study, the strength of evidence that mastitis can or cannot cause PL in dairy cows is very low because the research methods used were not appropriate to test the hypothesis that mastitis can cause PL in dairy cows.

\section{CONCLUSIONS}

Our systematic review revealed that epidemiologic evidence that mastitis can cause PL in dairy cows was limited to 2 studies that used objective research methods (Risco et al., 1999; Moore et al., 2005). Four additional studies reported an association between mastitis and PL, but the study results were inconclusive because the exposure risk period for mastitis overlapped with the follow-up period for PL in study cows. Further research is needed (1) to support the hypothesis that mastitis in combination with low body condition score (or other exposure factors) can increase the risk of PL, (2) to compare the effect of clinical versus subclinical mastitis on PL, (3) to compare the effect of mastitis before and during gestation on PL, and (4) to compare the effect of mastitis on PL in dairy cows during different lactations.

\section{ACKNOWLEDGMENTS}

This study was funded in part with funds from the University of Florida's College of Veterinary Medicine.

\section{REFERENCES}

Britt, J. H. 2008. Oocyte development in cattle: Physiological and genetic aspects. Rev. Bras. Zootec. 37:110-115.

Chebel, R. C., J. E. Santos, J. P. Reynolds, R. L. Cerri, S. O. Juchem, and M. Overton. 2004. Factors affecting conception rate after artificial insemination and pregnancy loss in lactating dairy cows. Anim. Reprod. Sci. 84:239-255.

Cullor, J. S. 1990. Mastitis and its influences upon reproductive performance in dairy cattle. Pages 176-180 in Proc. Int. Symp. Bovine Mastitis, Indianapolis, IN. National Mastitis Council, Madison, WI, and American Association of Bovine Practitioners, Auburn, AL.

De Vries, A. 2006. Economic value of pregnancy in dairy cattle. J. Dairy Sci. 89:3876-3885.

Hansen, P. J., P. Soto, and R. P. Natzke. 2004. Mastitis and fertility in cattle - possible involvement of inflammation or immune activation in embryonic mortality. Am. J. Reprod. Immunol. 51:294-301.

Hernandez, J. A., C. A. Risco, F. S. Lima, and J. E. Santos. 2012. Observed and expected combined effects of clinical mastitis and low body condition on pregnancy loss in dairy cows. Theriogenology $77: 115-121$.

Hockett, M. E., F. M. Hopkins, M. J. Lewis, A. M. Saxton, H. H. Dowlen, S. P. Oliver, and F. N. Schrick. 2000. Endocrine profiles of dairy cows following experimentally induced clinical mastitis during early lactation. Anim. Reprod. Sci. 58:241-251.

Hoeben, D., C. Burvenich, E. Trevisi, G. Bertoni, J. Hamann, R. M. Bruckmaier, and J. W. Blum. 2000. Role of endotoxin and TNFalpha in the pathogenesis of experimentally induced coliform mastitis in periparturient cows. J. Dairy Res. 67:503-514.

Lee, J. I., and I. H. Kim. 2007. Pregnancy loss in dairy cows: The contributing factors, the effects on reproductive performance and the economic impact. J. Vet. Sci. 8:283-288.

Liberati, A., D. G. Altman, J. Tetzlaff, C. Mulrow, P. C. Gøtzsche, J. P. A. Ioannidis, M. Clarke, P. J. Devereaux, J. Kleijnen, and D. Moher. 2009. The PRISMA statement for reporting systematic reviews and meta-analyses of studies that evaluate healthcare interventions: Explanation and elaboration. BMJ 339:b2700.

López-Gatius, F., P. Santolaria, J. Yániz, J. Rutllant, and M. LópezBéjar. 2002. Factors affecting pregnancy loss from gestation Day 38 to 90 in lactating dairy cows from a single herd. Theriogenology $57: 1251-1261$. 
McDougall, S., F. M. Rhodes, and G. Verkerk. 2005. Pregnancy loss in dairy cattle in the Waikato region of New Zealand. N. Z. Vet. J. 53:279-287.

Moher, D., A. Liberati, J. Tetzlaff, D. G. Altman, and P. Group. 2009. Preferred reporting items for systematic reviews and metaanalyses: The PRISMA statement. PLoS Med. 6:e1000097.

Moore, D. A., M. W. Overton, R. C. Chebel, M. L. Truscott, and R. H. BonDurant. 2005. Evaluation of factors that affect embryonic loss in dairy cattle. J. Am. Vet. Med. Assoc. 226:1112-1118.

Paape, M. J., P. M. Rautiainen, E. M. Lilius, C. E. Malstrom, and T. H. Elsasser. 2002. Development of anti-bovine TNF-alpha mAb and ELISA for quantitating TNF-alpha in milk after intramammary injection of endotoxin. J. Dairy Sci. 85:765-773.

Persson Waller, K., I. G. Colditz, S. Lun, and K. Ostensson. 2003. Cytokines in mammary lymph and milk during endotoxin-induced bovine mastitis. Res. Vet. Sci. 74:31-36.

Pinedo, P. J., P. Melendez, J. A. Villagomez-Cortes, and C. A. Risco. 2009. Effect of high somatic cell counts on reproductive performance of Chilean dairy cattle. J. Dairy Sci. 92:1575-1580.

Rainard, P., and M. J. Paape. 1997. Sensitization of the bovine mammary gland to Escherichia coli endotoxin. Vet. Res. 28:231-238.

Rambeaud, M., R. A. Almeida, G. M. Pighetti, and S. P. Oliver. 2003. Dynamics of leukocytes and cytokines during experimentally induced Streptococcus uberis mastitis. Vet. Immunol. Immunopathol. 96:193-205.

Ribeiro, E. S., G. Gomes, L. F. Greco, R. L. Cerri, A. Vieira-Neto, P. L. Monteiro Jr., F. S. Lima, R. S. Bisinotto, W. W. Thatcher, and
J. E. Santos. 2016. Carryover effect of postpartum inflammatory diseases on developmental biology and fertility in lactating dairy cows. J. Dairy Sci. 99:2201-2220.

Risco, C. A., G. A. Donovan, and J. Hernandez. 1999. Clinical mastitis associated with abortion in dairy cows. J. Dairy Sci. 82:1684-1689.

Samadzadeh, G. R., T. Rigi, and A. R. Ganjali. 2013. Comparison of four search engines and their efficacy with emphasis on literature research in addiction (prevention and treatment). Int. J. High Risk Behav. Addict. 1:166-171.

Santos, J. E., R. L. Cerri, M. A. Ballou, G. E. Higginbotham, and J. H. Kirk. 2004. Effect of timing of first clinical mastitis occurrence on lactational and reproductive performance of Holstein dairy cows. Anim. Reprod. Sci. 80:31-45.

Schams, D., and B. Berisha. 2004. Regulation of corpus luteum function in cattle-An overview. Reprod. Domest. Anim. 39:241-251.

Shuster, D. E., M. E. Kehrli Jr., and M. G. Stevens. 1993. Cytokine production during endotoxin-induced mastitis in lactating dairy cows. Am. J. Vet. Res. 54:80-85.

Soto, P., R. P. Natzke, and P. J. Hansen. 2003. Identification of possible mediators of embryonic mortality caused by mastitis: Actions of lipopolysaccharide, prostaglandin F2alpha, and the nitric oxide generator, sodium nitroprusside dihydrate, on oocyte maturation and embryonic development in cattle. Am. J. Reprod. Immunol. 50:263-272.

Townson, D. H., and J. L. Pate. 1994. Regulation of prostaglandin synthesis by interleukin-1 beta in cultured bovine luteal cells. Biol. Reprod. 51:480-485. 